\title{
INVOLUTIONS WTTH FIXED POINT SET OF CONSTANT CODIMENSION
}

\begin{abstract}
KOICHI IWATA
ABSTRACT.The cobordism classes of manifolds admitting involutions with fixed point set of codimension 5 are determined by means of Stiefel-Whitney classes.
\end{abstract}

1. Introdaction. Let $\mathfrak{R}_{n}$ be the group of nonoriented cobordism classes of $n$-dimensional smooth manifolds and let $J_{n}^{k}$ be its subset consisting of the classes which are represented by manifolds admitting smooth involutions with fixed point set of constant codimension $k . J_{n}^{k}$ is a subgroup of $\Re_{n}$ and $J_{*}^{k}=\sum_{n=k}^{\infty} J_{n}^{k}$ is an ideal of the nonoriented cobordism ring $\mathfrak{R}_{*}=\sum_{n=0}^{\infty} \mathfrak{N}_{n}$. Capobianco [1] proved the following results:

Proposition 1. $J_{n}^{3}$ is the set of classes $\alpha$ in $\Re_{n}$ with $W_{1}^{j} W_{n-j}(\alpha)=W_{1}^{i-5} W_{n-i} s_{5}(\alpha)$ $=0$, for each $i, j, 0 \leqslant j \leqslant n, 5 \leqslant i \leqslant n$.

Proposition 2. $J_{*}^{5} \subset J_{*}^{3}$.

In this note, we shall prove

THEOREM. $J_{n}^{5}$ is the set of classes $\alpha$ in $J_{*}^{3}$ with $W_{1}^{n-8} W_{2}^{4}(\alpha)=W_{1}^{n-9} W_{2}^{3} W_{3}(\alpha)=$ $W_{1}^{n-10} W_{2}^{2} W_{3}^{2}(\alpha)=W_{1}^{n-11} W_{2} W_{3}^{3}(\alpha)=W_{1}^{n-12} W_{3}^{4}(\alpha)=0$.

2. Characteristic numbers of classes in $J_{*}^{5}$. Let $\xi \rightarrow V$ be a smooth $k$-plane bundle over a closed smooth manifold $V$ and let $\pi: R P(\xi) \rightarrow V$ be the associated projective. space bundle. Denote by $a$ the characteristic class of the canonical line bundle $\lambda \rightarrow R P(\xi)$. Then by $[2, \S 21], H^{*}\left(R P(\xi) ; Z_{2}\right)$ is the free $H^{*}\left(V ; Z_{2}\right)$-module via $\pi^{*}$ on the classes $1, a, \ldots, a^{k-1}$, subject to the relation $\sum_{j=0}^{k} a^{k-j} \pi^{*}\left(v_{j}\right)=0$, where $v_{j}$ is the $j$ th Whitney class of $\xi$. The total Stiefel-Whitney class of $R P(\xi)$ is given by

$$
W(R P(\xi))=\pi^{*}(W(V))\left(\sum_{j=0}^{k}(1+a)^{k-j} \pi^{*}\left(v_{j}\right)\right) .
$$

Now suppose that a class $\alpha$ is represented by a manifold $M^{n}$ admitting an involution with fixed point set $F$ of codimension $k$. Let $q: \nu \rightarrow F$ be the normal bundle. Then by [2, (22.2)], $\alpha$ is the class of $R P(\nu \oplus R)$, which is the total space of the projective space bundle associated to $p: \nu \oplus R \rightarrow F$. Let $e$, resp. $c$, be the characteristic class of the canonical line bundle $\lambda \rightarrow R P(\nu \oplus R)$, resp. $\lambda \rightarrow R P(\nu)$.

Received by the editors March 26, 1981.

1980 Mathematics Subject Classification. Primary 57R85; Secondary 57R90. 
Then we have

Proposition 3. For any $x \in H^{j}\left(F ; Z_{2}\right), 0<j<n-k$,

$$
\left\langle p^{*}(x) e^{n-j},[R P(\nu \oplus R)]\right\rangle=\left\langle q^{*}(x) c^{n-1-j},[R P(\nu)]\right\rangle \text {. }
$$

The proof can be found in [5]. It follows from [2, §25]

Proposition 4. If $q^{*}(x) c^{n-1-j}$ represents a characteristic class of $\lambda \rightarrow R P(\nu)$, then $\left\langle p^{*}(x) e^{n-j},[R P(\nu \oplus R)]\right\rangle=0$.

Let us apply these facts to the case of constant codimension 5.

LEMMA 5. If $\alpha \in J_{*}^{5}$, then $W_{1}^{n-8} W_{2}^{4}(\alpha)=W_{1}^{n-9} W_{2}^{3} W_{3}(\alpha)=W_{1}^{n-10} W_{2}^{2} W_{3}^{2}(\alpha)=$ $W_{1}^{n-11} W_{2} W_{3}^{3}(\alpha)=W_{1}^{n-12} W_{3}^{4}(\alpha)=0$.

Proof. Denote $W_{j}=W_{j}(R P(\nu \oplus R)), W_{j}^{\prime}=W_{j}(R P(\nu))$ and let $v_{j}$, resp. $w_{j}$, be the $j$ th Whitney class of $\nu$, resp. $F$. By Propositions 3 and 4 ,

$$
\left\{\begin{array}{l}
W_{1}^{n-8} W_{2}^{4}=p^{*}\left(w_{1}+v_{1}\right)^{n-8} e^{8} \\
\left(W_{1}^{\prime}+c\right)^{n-8} c^{7}=q^{*}\left(w_{1}+v_{1}\right)^{n-8} c^{7}
\end{array}\right.
$$

give $W_{1}^{n-8} W_{2}^{4}(\alpha)=0$. The other relations can be obtained by the following computations:

$$
\begin{aligned}
& \left\{\begin{array}{c}
W_{1}^{n-9} W_{2}^{3} W_{3}=p^{*}\left\{\left(w_{1}+v_{1}\right)^{n-9} w_{1}\right\} e^{8}, \\
\left(W_{1}^{\prime}+c\right)^{n-9}\left\{\left(W_{2}^{\prime}+W_{1}^{\prime} c\right)\left(W_{3}^{\prime}+W_{2}^{\prime} c\right) c^{3}+\left(W_{1}^{\prime}+c\right)\left(W_{2}^{\prime}+W_{1}^{\prime} c\right)^{2} c^{3}\right. \\
=q^{*}\left\{\left(w_{1}+v_{1}\right)^{n-9} w_{1}\right\} c^{7},
\end{array}\right. \\
& \left\{\begin{array}{c}
W_{1}^{n-10} W_{2}^{2} W_{3}^{2}=p^{*}\left\{\left(w_{1}+v_{1}\right)^{n-10} w_{1}^{2}\right\} e^{8}, \\
\left(W_{1}^{\prime}+c\right)^{n-10}\left\{W_{1}^{\prime 2} W_{2}^{\prime 2} c^{3}+W_{3}^{\prime 2} c^{3}+W_{2}^{\prime 4} c\right\}=q^{*}\left\{\left(w_{1}+v_{1}\right)^{n-10} w_{1}^{2}\right\} c^{7},
\end{array}\right. \\
& \left\{\begin{array}{c}
W_{1}^{n-11} W_{2} W_{3}^{3}=p^{*}\left\{\left(w_{1}+v_{1}\right)^{n-11} w_{1}^{3}\right\} e^{8}, \\
\left(W_{1}^{\prime}+c\right)^{n-11}\left\{W_{2}^{\prime 2} W_{3}^{\prime 2}+W_{2}^{\prime 3} W_{3}^{\prime} c+\left(W_{2}^{\prime 4}+W_{1}^{\prime 2} W_{3}^{\prime 2}+W_{1}^{\prime} W_{2}^{\prime 2} W_{3}^{\prime}\right) c^{2}\right.
\end{array}\right. \\
& =q^{*}\left\{\left(w_{1}+v_{1}\right)^{n-11} w_{1}^{3}\right\} c^{7}, \\
& \left\{\begin{array}{c}
W_{1}^{n-12} W_{3}^{4}=W^{*}\left\{\left(w_{1}+v_{1}^{\prime 3}+W_{1}^{\prime} W_{3}^{\prime 2}\right) c^{3}\right\} \\
\left(W_{1}^{\prime}+c\right)^{n-12} W_{2}^{\prime 4} c^{3}=q^{*}\left\{\left(w_{1}+v_{1}\right)^{n-12} w_{1}^{4}\right\} c^{7} .
\end{array}\right.
\end{aligned}
$$

3. A system of generators of $J_{*}^{5}$. As is well known, $\mathfrak{R}_{*}$ is a graded polynomial algebra over $Z_{2}$ with one generator in each dimension $n$ which is not of the form $2^{r}-1$. We shall choose a suitable system of generators of $\mathfrak{R}_{*}$ for our purpose. Let $\left(n_{1}, n_{2}, \ldots, n_{2 k}\right)$ be a $2 k$-tuple of nonnegative integers with $n_{1}+n_{2}+\cdots+n_{2 k}$ $=n-2 k+1$. We denote by $R P\left(n_{1}, n_{2}, \ldots, n_{2 k}\right)$ the projective space bundle associated to the bundle $\lambda_{1} \oplus \lambda_{2} \oplus \cdots \oplus \lambda_{2 k}$ over $\operatorname{RP}\left(n_{1}\right) \times \operatorname{RP}\left(n_{2}\right)$ $\times \cdots \times R P\left(n_{2 k}\right)$, where $\lambda_{i}(i=1,2, \ldots, 2 k)$ is the pull-back of the canonical line bundle over the $i$ th factor. Stong [4, Lemma 3.4] proved that 
$R P\left(n_{1}, n_{2}, \ldots, n_{2 k}\right)$ belongs to $J_{n}^{k}$ and is indecomposable in $\mathfrak{R}_{*}$ if and only if

$$
\left(\begin{array}{c}
n-1 \\
n_{1}
\end{array}\right)+\left(\begin{array}{c}
n-1 \\
n_{2}
\end{array}\right)+\cdots+\left(\begin{array}{c}
n-1 \\
n_{2 k}
\end{array}\right)
$$

is odd. First, we shall show

LEMMA 6. For each $n \geqslant 13$, not of the form $2^{r}$ or $2^{r}-1$, there exists a generator $u_{n} \in J_{n}^{5}$ which is indecomposable in $\Re_{*}$.

Proof. If $\left(\begin{array}{c}n-1 \\ n-9\end{array}\right) \equiv 0 \bmod 2, R P(n-9,0, \ldots, 0)(9$ zeroes) is indecomposable in $\mathfrak{N}_{*}$. Consider the case $\left(\begin{array}{c}n-1 \\ n-9\end{array}\right) \equiv 1 \bmod 2$. Let $n-1=2^{r_{1}}+2^{r_{2}}+\cdots+2^{r_{1}}, r_{1}>r_{2}$ $>\cdots>r_{t} \geqslant 0$. Since $\left(\begin{array}{c}n-1 \\ n-9\end{array}\right)=\left(\begin{array}{c}n-1 \\ 8\end{array}\right), \quad\left\{r_{1}, r_{2}, \ldots, r_{t}\right\}$ contains 3. When $\left\{r_{1}, r_{2}, \ldots, r_{t}\right\}$ does not contain $1, R P(n-11,1,1,0, \ldots, 0)(7$ zeroes $)$ is indecomposable in $\mathfrak{N}_{*}$. When $\left\{r_{1}, r_{2}, \ldots, r_{t}\right\}$ contains 1 but does not contain 2, we can choose $R P(n-13,2,2,0, \ldots, 0)(7$ zeroes) as an indecomposable generator of $\mathfrak{N}_{*}$. Finally, suppose that $\left\{r_{1}, r_{2}, \ldots, r_{t}\right\}$ contains both 1 and 2 . Since $n-1$ is not of the form $2^{r}-1$ or $2^{r}-2$, there exists a number $i$ such that $r_{i}>r_{i+1}+1$. Then, $R P\left(2^{r_{1}}+\cdots+2^{r_{i}}-2,2^{r_{i+1}}+\cdots+2^{r_{t}}-14,8,0, \ldots, 0\right)(7$ zeroes $)$ is indecomposable in $\mathfrak{R}_{*}$.

Let $x_{2}$ be the class of $R P(2)$ and let $x_{2^{n}}$ be the class of $R P\left(2^{n}\right) \cup R P(2)^{n}$ for $n>1$. Denote by $y_{n}(n=5,6)$ the class of $R P(n-3,0,0,0)$ and by $z_{n}(n=$ $9,10,12)$ the class of $R P(n-5,0,0,0,0,0)$. Furthermore, by [3, $\S 7$, Remark] we know that there exists a class $z_{11}$ of an indecomposable manifold which belongs to $J_{11}^{5}$. Thus we have

LEMMA 7. $\mathfrak{R}_{*}$ is a polynomial algebra over $Z_{2}$ with the system of generators: $\left\{x_{2^{n}}\right.$ $\left.(n=1,2, \ldots), u_{n}\left(n \geqslant 13, n \neq 2^{r}, 2^{r}-1\right), y_{5}, y_{6}, z_{9}, z_{10}, z_{11}, z_{12}\right\}$.

Now we shall go into $J_{n}^{5}$. By direct computations as in Lemma 5 , we have

LEMMA 8. If $n \leqslant 10$ or $n=12$, then $s_{n}(\alpha)=0$ for any $\alpha \in J_{n}^{5}$.

Moreover, we have

LemMa 9. Let $n=2^{s}, s \geqslant 4$. Then $J_{n}^{5}$ contains a class $\alpha$ such that $s_{2^{-1}, 2^{-1}}(\alpha) \equiv 1$ $\bmod 2$.

Proof. For $s=4, \operatorname{RP}(7,0, \ldots, 0)$ (9 zeroes) is as required. For $s>4$,

$$
R P\left(2^{s-3}-2, s^{s-3}-1, \ldots, 2^{s-3}-1,0,0\right)
$$

is as required.

Let us observe monomials of the generators for $\mathfrak{N}_{*}$. First, notice that $J_{*}^{3} \subset J_{*}^{2}$ follows from [4] and Proposition 1. By their definitions, $y_{5}, y_{6} \in J_{*}^{2}$ and $z_{9}, z_{10}, z_{12}$ $\in J_{*}^{3}$. Furthermore, Proposition 1 shows $y_{5} y_{6}, x_{4}^{2} \in J_{*}^{3}$ and Lemma 9 shows $x_{2^{r}}^{2} \in J_{*}^{5}$ for $r \geqslant 3$. Clearly, $y_{5}^{2} \in J_{*}^{5}$. Now consider $y_{6}^{2}$. By the examination of the characteristic numbers, we can see that $y_{6}^{2}$ is the class $x_{2} y_{5}^{2}+\{\operatorname{RP}(3,2,2,0,0,0)\}$. Recall that $R P(\lambda)=R P(3,2,2,0,0,0)$ is the projective space bundle over $M=$ $R P(3) \times R P(2)^{2} \times R P(0)^{3}$ associated to $\lambda=\lambda_{1} \oplus \lambda_{2} \oplus \cdots \oplus \lambda_{6} \rightarrow M$. An involution of $M$, given by $\left(a, b_{1}, b_{2}, c_{1}, c_{2}, c_{3}\right) \rightarrow\left(a, b_{2}, b_{1}, c_{1}, c_{2}, c_{3}\right)$, induces a fiber 
preserving involution $T$ of $R P(\lambda)$; i.e., we can define an involution $T$ of $R P(\lambda)$ by

$$
T\left(u_{1}, u_{2}, u_{3}, u_{4}, u_{5}, u_{6}\right)=\left(-u_{1},-u_{3},-u_{2},-u_{4}, u_{5}, u_{6}\right) \text {. }
$$

It is easy to see that all the components of the fixed point set of $T$ are of codimension 5. Therefore $y_{6}^{2} \in J_{*}^{5}$. Referring to the results of [1], we can show that $J_{*}^{5}$ contains all monomials of generators for $J_{*}^{3}$ except those of the form

$$
y_{5} y_{6} x(m), \quad z_{9} x(m), \quad x_{4}^{2} x(m), \quad z_{10} x(m), \quad z_{12} x(m) \text {. }
$$

Here, $x(m)$ is the class of $R P\left(2^{r_{1}}\right) \times R P\left(2^{r_{2}}\right) \times \cdots: \times R P\left(2^{r_{1}}\right)$ for $m=2^{r_{1}}+2^{r_{2}}$ $+\cdots+2^{r_{t}}, r_{1}>r_{2}>\cdots>r_{t}>0$. By straightforward calculation, we have the tables of characteristic numbers.

\begin{tabular}{|c|c|c|c|}
\hline & $x_{4}^{2} x(n-8)$ & $z_{10} x(n-10)$ & $z_{12} x(n-12)$ \\
\hline$W_{1}^{n-8} W_{2}^{4}$ & $1 \quad i^{\prime}$ & $\begin{array}{l}0 \quad n \equiv 0,4(8) \\
1 \quad n \equiv 2,6(8)\end{array}$ & $\begin{array}{ll}0 & n \equiv 0,2(8) \\
1 & n \equiv 4,6(8)\end{array}$ \\
\hline$W_{1}^{n-10} W_{2}^{2} W_{3}^{2}$ & 1 & $\begin{array}{ll}1 & n \equiv 0,4(8) \\
0 & n \equiv 2,6(8)\end{array}$ & $\begin{array}{ll}1 & n \equiv 0,6(8) \\
0 & n \equiv 2,4(8)\end{array}$ \\
\hline$W_{1}^{n-12} W_{3}^{4}$ & 1 & $\begin{array}{ll}0 & n \equiv 0,4(8) \\
1 & n \equiv 2,6(8)\end{array}$ & $\begin{array}{ll}1 & n \equiv 0,2(8) \\
0 & n \equiv 4,6(8)\end{array}$ \\
\hline
\end{tabular}

\begin{tabular}{l|c|c} 
& $z_{9} x(n-9)$ & $y_{5} y_{6} x(n-11)$ \\
\hline$W_{1}^{n-9} W_{2}^{3} W_{3}$ & 1 & 0 \\
\hline$W_{1}^{n-11} W_{2} W_{3}^{3}$ & & 1
\end{tabular}

Using these, together with Lemmas 5, 7 and 8, we can attain our theorem immediately.

REMARK. As a corollary, we can show $J_{*}^{2 k+1} \subset J_{*}^{5}$ for every integer $k \geqslant 3$.

\section{REFERENCES}

1. F. L. Capobianco, Cobordism classes represented by fiberings with fiber $R P(2 k+1)$, Michigan Math. J. 24 (1977), 185-192. MR 57\# 1514.

2. P. E. Conner, Differentiable periodic maps, 2nd ed., Lecture Notes in Math., vol. 738, SpringerVerlag, Berlin and New York, 1979.

3. C. Kosniowski and R. E. Stong, Involutions and characteristic numbers, Topology 17 (1978), 309-330.

4. R. E. Stong, On fibering of cobordism classes, Trans. Amer. Math. Soc. 178 (1973), 431-447. MR 47\#4282.

5. T. Wada, Manifolds with involution whose fixed point set has codimension six, Bull. Yamagata Univ. Natur. Sci. 10 (1981), 193-205.

College of General Education, Tôhoxu Universtry, SendaI, Japan 